\title{
Squeezing water from a stone: High-throughput sequencing from a 145-year old holotype resolves (barely) a cryptic species problem in flying lizards
}

\author{
Jimmy A McGuire ${ }^{\text {Corresp.. }}$ 1, 2 ， Darko D Cotoras ${ }^{3,4}$ ， Brendan O'Connell ${ }^{5}$ ， Shobi Z S Lawalata ${ }^{1,2,6}$, Cynthia Y Wang- \\ Claypool ${ }^{1,2}$, Alexander Stubbs ${ }^{1,2}$, Xiaoting Huang ${ }^{7}$, Guinevere $\mathbf{O}$ U Wogan ${ }^{8}$, Sarah M Hykin ${ }^{1,2}$, Sean B \\ Reilly $^{1,2}$, Ke Bi ${ }^{9}$, Awal Riyanto ${ }^{10}$, Evy Arida ${ }^{10}$, Lydia L Smith ${ }^{1}$, Heather Milne ${ }^{5}$, Jeffrey W Streicher ${ }^{11}$, \\ Djoko T Iskandar ${ }^{12}$ \\ 1 Museum of Vertebrate Zoology, University of California, Berkeley, Berkeley, California, United States \\ 2 Department of Integrative Biology, University of California, Berkeley, Berkeley, California, United States \\ 3 Department of Ecology and Evolutionary Biology, University of California, Santa Cruz, Santa Cruz, California, United States \\ 4 Entomology Department, California Academy of Sciences, San Francisco, California, United States \\ 5 Department of Biomolecular Engineering and Bioinformatics, Baskin School of Engineering, University of California, Santa Cruz, Santa Cruz, California, \\ United States \\ 6 Jalan Hayam Wuruk, United in Diversity Foundation, Jakarta, Indonesia \\ 7 Key Laboratory of Marine Genetics and Breeding, Ocean University of China, Qingdao, China \\ 8 Department of Environmental Science, Policy, and Management, University of California, Berkeley, Berkeley, California, United States \\ ${ }^{9}$ Computational Genomics Resource Laboratory, California Institute for Quantitative Biosciences, University of California, Berkeley, Berkeley, California, \\ United States \\ 10 Museum Zoologicum Bogoriense (MZB), Indonesia Institute of Sciences (LIPI), Cibinong, West Java, Indonesia \\ 11 Department of Life Sciences, The Natural History Museum, London, London, United Kingdom \\ 12 School of Life Sciences and Technology, Institute of Technology Bandung, Bandung, West Java, Indonesia \\ Corresponding Author: Jimmy A McGuire \\ Email address: mcguirej@berkeley.edu
}

We used Massively Parallel High-Throughput Sequencing to obtain genetic data from a 145-year old holotype specimen of the flying lizard, Draco cristatellus. Obtaining genetic data from this holotype was necessary to resolve an otherwise intractable taxonomic problem involving the status of this species relative to closely related sympatric Draco species that cannot otherwise be distinguished from one another on the basis of museum specimens. Initial analyses suggested that the DNA present in the holotype sample was so degraded as to be unusable for sequencing. However, we used a specialized extraction procedure developed for highly degraded ancient DNA samples and MiSeq shotgun sequencing to obtain just enough low-coverage mitochondrial DNA (547 base pairs) to conclusively resolve the species status of the holotype as well as a second known specimen of this species. The holotype was prepared before the advent of formalin-fixation and therefore was most likely originally fixed with ethanol and never exposed to formalin. Whereas conventional wisdom suggests that formalin-fixed samples should be the most challenging for DNA sequencing, we propose that evaporation during long-term alcohol storage and consequent water-exposure may subject older ethanol-fixed museum 
specimens to hydrolytic damage. If so, this may pose an even greater challenge for sequencing efforts involving historical samples. 
1 Squeezing water from a stone: High-Throughput Sequencing from a 145-year old holotype 2 resolves (barely) a cryptic species problem in flying lizards

3

4

Jimmy A. McGuire ${ }^{1,2}$, Darko D. Cotoras ${ }^{3,4}$, Brendan O’Connell ${ }^{5}$, Shobi Z. S. Lawalata ${ }^{1,2,6}$, Cynthia Y. Wang-Claypool ${ }^{1,2}$, Alexander Stubbs ${ }^{1,2}$, Xiaoting Huang ${ }^{7}$, Guinevere O. U. Wogan ${ }^{8}$, Sarah M. Hykin ${ }^{1,2}$, Sean B. Reilly ${ }^{1,2}, \mathrm{Ke} \mathrm{Bi}^{9}$, Awal Riyanto ${ }^{10}$, Evy Arida ${ }^{10}$, Lydia L. Smith ${ }^{1}$, Heather Milne ${ }^{5}$, Jeffrey W. Streicher ${ }^{11}$, and Djoko T. Iskandar ${ }^{12}$.

${ }^{1}$ Museum of Vertebrate Zoology, 3101 VLSB, University of California, Berkeley, CA 94720, USA

${ }^{2}$ Department of Integrative Biology. University of California, Berkeley, CA 94720, USA

${ }^{3}$ Department of Ecology and Evolutionary Biology, University of California, Santa Cruz, 1156 High Street, Santa Cruz, CA 95064, USA

${ }^{4}$ Entomology Department, California Academy of Sciences, 55 Music Concourse Dr., Golden Gate Park, San Francisco, CA 94118, USA

${ }^{5}$ Department of Biomolecular Engineering and Bioinformatics, Baskin School of Engineering, University of California, Santa Cruz, 1156 High Street, Santa Cruz, CA 95064, USA

${ }^{6}$ United in Diversity Foundation, Jalan Hayam Wuruk No. 28, Jakarta 10220, Indonesia

${ }^{7}$ Key Laboratory of Marine Genetics and Breeding, Ocean University of China, Ministry of Education, 5 Yushan Road, Qingdao 266003, China

${ }^{8}$ Department of Environmental Science, Policy, and Management, University of California, Berkeley, CA 94720, USA

${ }^{9}$ Computational Genomics Resource Laboratory, California Institute for Quantitative Biosciences, 238 Koshland Hall, University of California, Berkeley, CA 94720, USA

${ }^{10}$ Museum Zoologicum Bogoriense (MZB), Indonesia Institute of Sciences (LIPI), Jalan Raya BogorJakarta KM 46, Cibinong 16911, Indonesia

${ }^{11}$ Department of Life Sciences, The Natural History Museum, London SW7 5BD, United Kingdom

${ }^{12}$ School of Life Sciences and Technology, Istitut Teknologi Bandung, Labtek XI Building, 10 Jalan Ganesa, Bandung 40132, Indonesia

Corresponding Author: Jimmy A. McGuire

Email address: mcguirej@berkeley.edu 
49

50

51

52

53

54

55

56

57

58

59

60

61

62

63

64

65

66

67

68

69

70

71

72

73

74

75

76

77

78

79

80

81

82

83

84

85

86

87

88

89

90

91

92

93

94

\begin{abstract}
We used Massively Parallel High-Throughput Sequencing to obtain genetic data from a 145year old holotype specimen of the flying lizard, Draco cristatellus. Obtaining genetic data from this holotype was necessary to resolve an otherwise intractable taxonomic problem involving the status of this species relative to closely related sympatric Draco species that cannot otherwise be distinguished from one another on the basis of museum specimens. Initial analyses suggested that the DNA present in the holotype sample was so degraded as to be unusable for sequencing. However, we used a specialized extraction procedure developed for highly degraded ancient DNA samples and MiSeq shotgun sequencing to obtain just enough low-coverage mitochondrial DNA (547 base pairs) to conclusively resolve the species status of the holotype as well as a second known specimen of this species. The holotype was prepared before the advent of formalin-fixation and therefore was most likely originally fixed with ethanol and never exposed to formalin. Whereas conventional wisdom suggests that formalin-fixed samples should be the most challenging for DNA sequencing, we propose that evaporation during long-term alcohol storage and consequent water-exposure may subject older ethanol-fixed museum specimens to hydrolytic damage. If so, this may pose an even greater challenge for sequencing efforts involving historical samples.
\end{abstract}

\title{
INTRODUCTION
}

The advent of Massively Parallel High-Throughput Sequencing (HTS) has dramatically altered the manner in which geneticists conduct their research. This is certainly true for molecular phylogeneticists and population geneticists, who now routinely have access to large multilocus genetic datasets for non-model organisms. Because HTS using the Illumina platform involves sequencing of small fragments of DNA, this approach offers the potential to access previously unattainable genome-scale sequence data even for degraded historical samples (e.g., Prüfer et al., 2014; Palkopoulou et al., 2015). Millions of fluid-preserved specimens in museum collections predate the development of allozyme and DNA sequencing technologies, and thus lack specially preserved tissue samples for genetic analysis. Formalin-fixed fluid specimens usually having highly fragmented and cross-linked DNA, are often refractory to sequencing efforts using traditional Sanger sequencing. However, recent studies have shown that it is possible to obtain genomic DNA sequences from some of these fluid-preserved museum specimens. Hykin, Bi \& McGuire (2015) demonstrated that low-coverage genomic sequences could be recovered from a 30 -year old formalin-fixed museum specimen, though they were unsuccessful with a $\sim 100$-year old specimen. Ruane and Austin (2016) sequenced Ultra-Conserved Elements (UCEs) from both formalin-fixed $(n=11)$ and ethanol-fixed $(n=10)$ museum specimens, including one sample that was collected between 1878 and 1911. Both had mixed success, with the quantity of DNA recovered in the extraction stage likely playing the largest role in the performance of their 
95

96

97

98

99

100

101

102

103

104

105

106

107

108

109

110

111

112

113

114

115

116

117

118

119

120

121

122

123

124

125

126

127

128

129

130

131

132

133

134

135

136

137

138

139

140

sequencing efforts. Notably, the samples that failed in Ruane and Austin's (2016) experiment included subsets of both their formalin- (7 of 16) and alcohol-fixed (4 of 5) samples, indicating that old alcohol-preserved museum specimens are not necessarily less problematic than those initially fixed with formalin. This is surprising given that contemporary tissue samples earmarked for genetic analysis are routinely stored in $95 \%$ ethanol.

Our objective in this study was to address an otherwise intractable problem in flying lizard taxonomy using Illumina HTS and ancient DNA methods for a 145 -year old fluidpreserved holotype specimen. The nettlesome taxonomic issue involves a small clade of poorly known flying lizards (genus Draco, Agamidae) that, for reasons outlined below, was unlikely to be resolved without obtaining genetic data from the holotype specimen of one of the constituent species, Draco cristatellus. Determining species limits within this small clade (the Draco fimbriatus group) has proven challenging for taxonomists, and we first describe the convoluted taxonomic history of the clade as a justification for our ultimate solution to this question involving HTS. The $D$. fimbriatus group currently includes four recognized species: $D$. abbreviatus, $D$. cristatellus, $D$. fimbriatus, and D. maculatus. This taxonomic framework is based on Manthey (2008) and was followed by the widely utilized Reptile Database (Uetz, 2006). For reasons that we will describe in a subsequent paper, we instead utilize an alternative taxonomy that includes $D$. cristatellus, as well as $D$. fimbriatus ( $=D$. abbreviatus above), $D$. hennigi, D. punctatus ( $=D$. fimbriatus above), and D. maculatus. We note that our taxonomy differs from that of Manthey (2008) primarily as a consequence of having information that indicates that the type locality of $D$. fimbriatus is the Malay Peninsula rather than Java. We further note that our recognition of both $D$. cristatellus and D. punctatus is tentative, as a primary objective of this paper is to resolve whether these are in fact distinct species.

The Draco fimbriatus group is taxonomically challenging. Although Draco maculatus is abundant, easily sampled in the field, and easily distinguished from other members of the group based on external phenotype, the remaining members of this clade are only rarely encountered, with relatively few specimens represented in museum collections. These species are canopy specialists (McGuire, 2003), making them more difficult to detect and more challenging to collect than other Draco taxa. Furthermore, the species comprising the D. fimbriatus group, as well as several other Draco clades are primarily distinguished on the basis of differences in coloration of their display structures (dewlap and patagia for most Draco taxa, just the dewlap among the relevant members of the $D$. fimbriatus group). For example, two major clades - the 'Philippines volans group' (McGuire and Alcala, 1999) and the 'Draco lineatus group' (McGuire et al., 2007) - are each composed of multiple species that are primarily distinguished on the basis of coloration. Because coloration fades in preservative, recognizing species-specific coloration characteristics generally requires experience with the species in the field and/or access to color imagery of the specimen in life. Thus, as museum specimens, the members of these clades are functionally cryptic sympatric species. In summary, for the $D$. fimbriatus group, the paucity of museum specimens, and the rarity with which specimens are observed in the field from throughout their collective ranges by single observers, has greatly impeded taxonomic progress.

Within the Draco fimbriatus group, a particularly challenging issue relates to the taxonomic standings of D. cristatellus Günther 1872 and D. punctatus Boulenger 1900. Draco cristatellus was described based on a single specimen collected by Mr. Alfred Hart Everett in Matang, Sarawak between 1869 and March of 1872 (when Günther's manuscript describing the species was submitted for publication). Although Everett collected the type specimen, the 
141 Trustees of the British Museum purchased it from Mr. W. Cutter, thereby making it available to 142 Günther for description (see Günther, 1872). Because Günther presumably did not see the living 143 specimen, he did not evaluate the coloration of the dewlap in life, which is essential for species 144 identification within this group. Günther described the dewlap as 'golden-yellow, with a brown

145

146

147

148

149

150

151

152

153

154

155

156

157

158

159

160

161

162

163

164

165

166

167

168

169

170

171

172

173

174

175

176

177

178

179

180

181

182

183

184

185

186 anterior edge', presumably from its preserved state. Subsequently, Boulenger (1900) described $D$. punctatus from Bukit Larut on the Malay Peninsula, noting that the dewlap was lemon yellow in coloration. Although he did not attempt to diagnose D. punctatus from D. cristatellus, Boulenger (1900) was clearly aware of the latter species and explicitly considered his $D$. punctatus holotype to be taxonomically distinct. Indeed, Boulenger (1900) noted that he had examined a second specimen of $D$. punctatus from Sarawak that was also collected by Everett, remarking that he had previously referred that second specimen to D. cristatellus. Boulenger (1900) might be the last author to have had a clear idea about the taxonomic distinctiveness of D. cristatellus and $D$. punctatus, and it is a pity that he did not identify the character differences that he used to render his taxonomic decision. Although de Rooij (1915) recognized D. fimbriatus, D. cristatellus and D. punctatus as distinct species, subsequent authors synonymized one or more members of the group. Hennig (1936) synonymized $D$. cristatellus with $D$. fimbriatus, while continuing to recognize D. punctatus. In his monographic Draco taxonomic study, Musters (1983) opted to synonymize both $D$. cristatellus and $D$. punctatus with D. fimbriatus. In his competing taxonomic treatment, Inger (1983) recognized two species, $D$. cristatellus and D. fimbriatus, as valid species, but placed $D$. punctatus in the synonymy of $D$. cristatellus. Inger's (1983) recognition of two species was based in part on Grandison's (1972) report on two sympatric $D$. fimbriatus group species with distinct dewlap colorations on Gunung (Mt.) Benom on the Malay Peninsula. Whereas Grandison (1972) identified the two sympatric species as D. fimbriatus and D. punctatus (without commenting on the status of the Bornean D. cristatellus), Inger (1983) instead opted to treat $D$. punctatus as a synonym of $D$. cristatellus. This sensible decision was presumably made on the basis of the similar dewlap colorations of the $D$. cristatellus and $D$. punctatus holotypes ('golden-yellow, with a brown anterior edge' vs. 'lemon yellow'). Inger (1983) furthermore attempted to differentiate his conceptions of $D$. fimbriatus and D. cristatellus using a statistical analysis of eight linear measurements and scale counts. Although he successfully sorted his sample into two groups on the basis of overlapping but significantly distinct character state differences, his a priori placement of $D$. punctatus in the synonymy of $D$. cristatellus effectively precluded the possibility that three species $-D$. cristatellus, $D$. fimbriatus, and D. punctatus - might all co-occur on the Greater Sunda Shelf (and particularly on Borneo). Here we address this open question taking advantage of two critical developments: (1) the acquisition and analysis of a key specimen (TNHC 56763) obtained by JAM from Santubong, Sarawak, Malaysian Borneo in 1996, and (2) an analysis of the 145-year old $D$. cristatellus holotype using ancient DNA extraction methods and HTS on the Illumina platform. We establish the following hypothesis-testing framework: In Hypothesis 1, D. cristatellus and D. punctatus are synonyms, together representing a single species distinct from $D$. fimbriatus and TNHC 56763. In Hypothesis 2, D. cristatellus and D. fimbriatus are synonyms. In Hypothesis 3 , $D$. cristatellus is a species distinct from $D$. punctatus and $D$. fimbriatus but conspecific with TNHC 56763. Finally, in Hypothesis 4, D. cristatellus, D. fimbriatus, D. punctatus, and TNHC 56763 all represent distinct species, with TNHC 56763 representing a fourth sympatric species on Borneo. The only way to conclusively test these alternative hypotheses is to obtain informative genetic data from the holotype specimen of $D$. cristatellus for comparison with TNHC 56763, as well as representative specimens of D. fimbriatus and D. punctatus. 
(a) DNA extraction and sequencing from the Draco cristatellus holotype

191 Draco cristatellus (specimen BMNH 1872.2.19.4). This specimen was originally collected and prepared prior to March 1872, well before the advent of formalin-fixation. At that time, the standard practice for fluid preservation of reptiles, amphibians, and fishes was direct preservation in "pure spirits of wine" (Günther, 1880). Thus, the holotype was most likely initially fixed in $90-100 \%$ ethanol (= Günther's "pure spirits of wine") and never exposed to formalin. Nevertheless, we opted to perform our initial DNA extraction using the methodology described in Hykin, Bi \& McGuire (2015) for formalin-fixed tissues, with the goal being to perform an exome-capture experiment with this sample. The Hykin, Bi \& McGuire (2015) procedure involves a series of initial ethanol washes followed by treatment in a heated alkali buffer solution to break cross-linkages before standard phenol-chloroform extraction. When this extraction returned a very low (potentially zero) yield, we performed a second pair of phenolchloroform extractions involving phase-lock gel tubes followed by SPRI bead clean-up. This second round of extractions was performed with and without exposure to heated alkali solution. These extractions also failed to return sufficient DNA to move forward with library preparation. Despite minimal DNA yield, we made an attempt to PCR-amplify and sequence a short fragment of the mitochondrial ND2 gene from both DNA extracts. These experiments resulted in the amplification and sequencing of human ND2 in two separate experiments. Both of our low-yield DNA extractions were then sent to MYcroarray Inc. in Ann Arbor, MI where they were subjected to an extra silica purification designed for low-concentration fragment retention, and prepared as libraries. However, the library preparation retrieved only artifact and we did not proceed to targeted enrichment of selected exons or sequencing.

At this stage, we engaged with a lab specializing in genetic analysis of ancient DNA samples, with extraction and sequencing performed in a facility specifically designed for work with ancient samples (the Shapiro Lab at UC Santa Cruz). No reptile work had previously been done in this facility and all work followed lab standards for working with historical samples (Fulton, 2011). The DNA extraction protocol was based on Dabney et al. (2013), Tin, Economo \& Mikheyev (2014), and D. D. Cotoras et al. (2017). An initial subsample of $40 \mathrm{mg}$ of tissue was subdivided into $\sim 1 \mathrm{~mm}$ pieces and suspended in lysis buffer. The composition of the $100 \mathrm{~mL}$ lysis buffer aliquot was: $5.3 \mathrm{~mL} \mathrm{1} \mathrm{M} \mathrm{Tris-} \mathrm{HCl}(\mathrm{pH} 8.0), 5.3 \mathrm{~mL}$ 0.2 M EDTA, $10.6 \mathrm{~mL} \mathrm{20 \%} \mathrm{Sarkosyl,}$ $1 \mathrm{~mL}$ 2-mercaptoethanol, and $77.8 \mathrm{~mL}$ distilled water. The tissue was digested with a total of 1 $\mathrm{mL}$ of lysis buffer with $1 \mathrm{mg} / \mathrm{mL}$ proteinase $\mathrm{K}$, initially incubated overnight at $56^{\circ} \mathrm{C}$, and then raised to $72^{\circ} \mathrm{C}$ for $1 \mathrm{hr}$. The $72^{\circ} \mathrm{C}$ incubation step was undertaken in case the sample had been exposed to formalin in order to reverse any potential crosslinks. Silica-based purification followed the centrifugation-based protocol described by Dabney et al. (2013). Briefly, $0.5 \mathrm{~mL}$ of $3 \mathrm{M}$ sodium acetate was added to the lysate and them transferred to a tube with $13 \mathrm{~mL}$ of binding buffer. The binding buffer is prepared in a $50 \mathrm{~mL}$ tube by first adding $23.88 \mathrm{~g}$ of guanidine hydrochloride and then adding water to bring the volume to $30 \mathrm{~mL}$. A key element of this purification protocol is the high salt concentration of this binding buffer, which enhances recovery of short DNA fragments. After complete dissolution of the guanidine hydrochloride, 25 $\mu \mathrm{l}$ of Tween-20 and sufficient isopropanol to bring the total volume to $50 \mathrm{~mL}$ were added. The mixture of sample, binding buffer, and sodium acetate was transferred into a Zymo extension 
233 reservoir attached to a MiniElute spin column. The spin column was then centrifuged for 10 234 minutes at 1,000 rpm, after which the spin column was transferred to a $1.5 \mathrm{~mL}$ Eppendorf tube. 235 We performed a dry spin for 1 minute at 13,000 rpm, followed by 2 washes with $750 \mu \mathrm{l}$ of PE 236 buffer (1 minute spin at 6,000 rpm). To ensure the entire PE buffer was removed, we did a dry 237 spin for 1 minute at maximum speed. We eluted the purified extract in two volumes of $25 \mu 1$ of 238 TET. Each sample was centrifuged for 30 seconds at 13,200 RPM after 3-5 minutes of incubation. Because the elution displayed pigmentation, $25 \mu$ of the extract was purified on a column filled with cross-linked polyvinylpyrrolidone (PVPP) (Arbeli and Fuentes, 2007). We also produced an extraction control consisting of lysis buffer that was subjected to the same set of procedures.

For genomic sequencing, we prepared three barcoded Illumina sequencing libraries (two for the holotype sample and one for the control) using the Meyer and Kircher (2010) protocol, starting with $5 \mu 1$ of the PVPP purified DNA extraction. The same volume was used for the extraction control. The libraries were sequenced on an Illumina MiSeq machine using 150-cycle v3 chemistry (2x75). Following sequencing, adaptors were removed from reads and sequences were merged using SeqPrep2 (https://github.com/jeizenga/SeqPrep2). Default parameters were used with the exception of the following: -q 20 -L 30 -A AGATCGGAAGAGCACACGTC -B AGATCGGAAGAGCGTCGTGT. FastQC (https://www.bioinformatics.babraham.ac.uk/ projects/fastqc/) confirmed that the sequence quality was good, with the normal base quality drop in the final five bases.

\section{(b) Sanger sequence data for Draco fimbriatus group specimens}

Our team has generated a large number of complete ND2 sequences for Draco specimens, including for 65 exemplars representing the $D$. fimbriatus group. These sequences were available for comparison with ND2 sequence fragments obtained from the $D$. cristatellus holotype. PCRamplification was undertaken using the primers Metf1 and ALAr2, with cycle sequencing involving these external primers plus the internal primers Metf5 and ND2r6 (see McGuire and Kiew 2001 for details).

\section{(c) Exome-capture and screening of mitochondrial DNA}

For another project (McGuire et al. in prep), we generated an exome-capture data set using the MyBaits in-solution capture system for a set of 350 samples spanning all of Draco. This sample set included 14 D. fimbriatus group samples. The target loci for the exome capture include 1400 exons and flanking sequences derived from transcriptome sequences (jointly representing 709 loci), which were supplemented with an additional 540 lizard-specific UCE loci. Libraries enriched for our target loci were barcoded and sequenced on an Illumina Hi Seq 4000. Although our experiment was specifically designed to avoid capturing mitochondrial genes, mitochondrial sequences are so abundant in genomic DNA extractions that some mitochondrial molecules inevitably find their way into the off-target by-catch (non-target DNA sequences that are obtained during an exome-capture experiment). We took advantage of this imperfect filter to obtain mitochondrial sequences for comparison with the $D$. cristatellus holotype. For TNHC 56763, we used Geneious version 8.1.7 (Kearse et al. 2012) to obtain a mostly complete representation of mitochondrial coding genes by mapping our raw exome capture data (including off-target sequences) to the complete mitochondrial genome of Acanthasaura armata available on GenBank (AB266452.1). A preliminary assessment of the identity of the sequences was performed with a BLAST search after collapsing duplicate sequences with fastx_collapser 
279 (http://hannonlab.cshl.edu/fastx toolkit/commandline.html\#fastx collapser usage). The result 280 of the BLAST search was visualized with the program MEGAN (Huson et al., 2007). Processed

281

282

283

284

285

286

287

288

289

290

291

292

293

294

295

296

297

298

299

300

301

302

303

304

305

306

307

308

309

310

311

312

313

314

315

316

317

318

319

320

321

322

323

324

reads were mapped with BWA mem (Li and Durbin, 2009) against the reference partial

mitochondrial genome of TNHC 56763. Duplicates were removed with samtools rmdup (Li et al., 2009). A total of 17 unique reads mapped against the reference after duplicate removal. The represent a total of $777 \mathrm{bp}$ of the $8114 \mathrm{bp}$ reference. Most of the mapped regions had 1x coverage and portions of four contigs had $2 \mathrm{x}$ coverage. The average length of the mapped reads was $53 \mathrm{bp}$. Finally, for each of the $14 \mathrm{D}$. fimbriatus group samples included in our exomecapture experiment, we used Geneious to map our raw sequencing reads to 10 mitochondrial contigs obtained for the $D$. cristatellus holotype. The raw sequence data is available on the SRA database.

\section{(d) Analysis of DNA sequence variation}

Our analysis of DNA sequence variation included alignment of homologous DNA sequences and a simple count of nucleotide base substitutions between the Draco cristatellus holotype, sample TNHC 56763 from Santubong, Sarawak, and our selection of D. fimbriatus and D. punctatus samples from the Malay Peninsula, Sumatra, the Mentawai Islands, Java, and Borneo. Specimens examined are listed in Table 1 . We also performed a heuristic parsimony analysis to obtain a phylogram for the $D$. fimbriatus group and performed a non-parametric bootstrap analysis with 1000 replicates to assess branch support. We did not perform a more rigorous maximum likelihood or Bayesian analysis because our primary objective was to assess uncorrected relative branch lengths. Phylogenetic analyses were performed in PAUP version 4 (Swofford 2002).

\section{(e) Data availability and Permits}

Sanger sequence data are available on GenBank (will be submitted if accepted) and a matrix for the mitochondrial ND2 gene for the D. fimbriatus group is included as supplemental materials. This research was undertaken in accordance with UC Berkeley Animal Use Protocol Number AUP-2014-12-6954. Fieldwork was undertaken with research permits issued by the Economic Planning Unit of Malaysia (UPE:40/200/19 SJ.363) and the Indonesia Institute of Sciences (LIPI: No. 2411/FRP/SM/X?2008 and No. 0115/FRP/SM/VI/2009).

\section{RESULTS}

The MiSeq run generated a total of 1,086,926 paired-end reads after combining the data from 2 different libraries (prepared identically) from the same extract. The average total library fragment length was $75 \mathrm{bp}$, whereas the average length of the mapped reads was $53 \mathrm{bp}$. Raw sequences were merged if possible and duplicates collapsed, producing a total of 538,995 reads, which were BLAST searched (Altschul et al. 1990) against the NCBI database. 115,266 reads were successfully assigned, and of these, 47,400 hits corresponded to bacteria, 40,751 were assigned to mammals (of which 29,720 were specifically assigned to human), 334 were assigned to Reptilia, and 48 were specifically assigned to Anolis carolinensis. Only three reads were assigned to Draco, each of which involved the mitochondrial ND2 sequence posted on GenBank for Draco cristatellus sample TNHC 56763 (see below). The paucity of Draco hits is not surprising given that there are no Draco reference genomes to map to. The extraction control was sequenced producing a total of 104,417 PE reads. After processing (adaptor removal, merged if possible, and duplicate removal) a total of 15,480 reads were assigned by the BLAST search. 
325 Bacteria were represented by 6,805 reads, mammals by 4,711 reads (of those, 3103 were 326 assigned to human, and reptiles were assigned no reads (one read was assigned to chicken 327 (Gallus gallus).

328 Of the three holotype ND2 reads, two were broadly overlapping, and the joint ND2 data obtained from the holotype totaled only $125 \mathrm{bp}$. To search for additional mitochondrial contigs in the holotype MiSeq data, we first generated a partial mitochondrial genome for TNHC 56763

331

332

333

334 from exome-capture off-target by-catch, which returned 8114 bp of protein-coding gene sequence data. Mapping the holotype data to the TNHC 56763 mitochondrial assembly resulted in the recovery of an additional 10 reads totaling $596 \mathrm{bp}$ of mitochondrial sequence data representing six genes (COI, COXIII, ATPase8, ND4, ND4L, ND5). Thus, a total of 13 contigs were assembled with an average length of $61 \mathrm{bp}$. Most of the mapped regions had 1x coverage and parts of four contigs had $2 \mathrm{x}$ coverage. No reads were recovered when mapping the extraction blank against the same reference. In comparing the holotype sequence data with TNHC 56763 across the $721 \mathrm{bp}$ of homologous sequence data, we found that the two samples were only weakly divergent from one another, sharing the same base calls at 710 of 721 positions for a raw sequence divergence of $1.5 \%$. We then mapped the raw reads from the exome captures for the remaining $13 \mathrm{D}$. fimbriatus group samples to the $10 \mathrm{D}$. cristatellus holotype contigs, which returned as few as two and as many as six homologous sequences per sample.

Comparison of the mitochondrial data obtained from the Draco cristatellus holotype with homologous data obtained for D. fimbriatus group samples found that the sample TNHC 56763 from Santubong, Sarawak, Malaysian Borneo was much more similar to the holotype than were any other $D$. fimbriatus group samples. When limiting our comparison to the six gene fragments for which we had between six and 13 corresponding sequences for comparison to the holotype, we found that TNHC 56763 was $0.9 \%$ divergent from the holotype, whereas all other samples ranged between $11.9 \%$ and $15.1 \%$ divergent. TNHC 56763 differed from the holotype at just five of 547 base positions, whereas the other samples differed from the holotype at from 36 of $303 \mathrm{bp}$ to 70 of 465 base positions. The ND2 comparisons were most comprehensive because we had access to complete ND2 sequences for 65 D. fimbriatus group samples. Whereas TNHC 56763 differed from the holotype at three of 183 base positions, all other samples differed by at least 24 base positions. Notably, the two D. punctatus samples from Sarawak (the type locality for $D$. cristatellus) differed from the holotype at 28 and 30 of 183 base positions $(15.3 \%$ and $16.4 \%$, respectively).

A parsimony phylogenetic analysis of the ND2 gene including the D. cristatellus holotype strongly supports the monophyly of the holotype together with TNHC 56763 to the exclusion of all other D. fimbriatus group samples with $100 \%$ bootstrap support (Figure 1).

360 Further, the 1.6\% ND2 sequence divergence between TNHC 56763 and the holotype is only slightly greater than that between samples of $D$. punctatus from a single locality (Sitahuis, Sumatra; uncorrected ND2 divergence of $1.2 \%$ ). This does not consider the possibility that one or more of the five documented base substitutions could be sequencing errors resulting either from damage to the holotype DNA or random errors in our low coverage data, as we did not apply informatics pipelines developed to identify post-mortem damage of ancient DNA to our data (e.g. Mateiu and Rannala 2008, Molak and Ho 2011). Notably, one of the three inferred ND2 substitutions is a first position $\mathrm{C}->\mathrm{T}$ change that would result in a proline to serine amino acid replacement, suggesting that this might be the result of post-mortem deamination of the template molecular or sequencing error. 


\section{DISCUSSION}

372

373

374

375

376

377

378

379

380

381

382

383

384

385

386

387

388

389

390

391

392

393

394

395

396

397

398

399

400

401

402

403

404

405

406

407

408

409

410

411

412

413

414

415

416

In the present study, we applied HTS to a 145-year old fluid-preserved holotype specimen in an effort to disentangle an otherwise intractable taxonomic question. The problem stems from the fact that one of the species in this group, Draco cristatellus, was described using limited color information, and because fluid-preserved specimens representing multiple sympatric $D$.

fimbriatus group species are often indistinguishable from one another without color information. Indeed, sympatric species in this complex are effectively cryptic once they have been prepared as museum specimens. This combination of circumstances rendered it virtually impossible to resolve the species status of $D$. cristatellus relative to D. punctatus and D. fimbriatus, two widespread species on the Greater Sunda Shelf. Importantly, a sample (TNHC 56763) collected in 1996 by JAM provides phylogenetic evidence for a third $D$. fimbriatus group species on Borneo, with the natural question being whether this sample is conspecific with the namebearing holotype specimen of $D$. cristatellus housed in the British Museum of Natural History. Several species composition outcomes were possible, all of which were considered in a hypothesis-testing framework. In Hypothesis $1, D$. cristatellus and D. punctatus are synonyms, together representing a single species distinct from $D$. fimbriatus and TNHC 56763. In Hypothesis 2, D. cristatellus and D. fimbriatus are synonyms. In Hypothesis $3, D$. cristatellus is a species distinct from D. punctatus and D. fimbriatus but conspecific with TNHC 56763. Finally, in Hypothesis 4, D. cristatellus, D. fimbriatus, D. punctatus, and TNHC 56763 all represent distinct species, with TNHC 56763 representing a fourth sympatric species on Borneo. The only way to conclusively test these alternative hypotheses was to obtain informative genetic data from the holotype specimen of D. cristatellus for comparison with TNHC 56763, and representative specimens of $D$. fimbriatus and D. punctatus.

We initially believed that genetic data would easily be retrieved from the Draco cristatellus holotype. The holotype was prepared before the advent of formalin-fixation, and we consequently had reason to believe that the specimen was originally fixed with ethanol and had never been exposed to formalin. Because tissue samples collected for genetic analysis are routinely stored in ethanol, we were confident that the holotype would still hold high molecularweight DNA suitable for genomic sequencing. Our hope was to perform exome-capture with this sample and include it in a larger Draco phylogenomic data set. However, our initial attempts at extracting DNA from the sample using methods appropriate for historical and formalin-fixed tissues failed, forcing us to adjust both our approach and our expectations. Fortunately, our alternative hypotheses proved testable without comprehensive genomic data from the holotype. Indeed, analysis of the initial $125 \mathrm{bp}$ of mitochondrial ND2 data identified when the holotype sequence data was subjected to GenBank BLAST allowed us to reject hypotheses 1,2, and 4 in favor of hypothesis 3 . The additional $422 \mathrm{bp}$ of mitochondrial data obtained via mapping of holotype contigs to the reconstructed mitochondrial genome for TNHC 56763 simply provided additional confirmation that $D$. cristatellus and D. punctatus are each valid species, and that our specimen TNHC 56763 from Santubong, Sarawak is indeed a true D. cristatellus exemplar. This finding was a best-case scenario because all future specimens for which tissue samples are obtained can now be compared with known D. cristatellus, D. punctatus, and D. fimbriatus samples for genetic identification.

What lessons can be learned from our attempt to obtain genetic data from the Draco cristatellus holotype? First, even when initial attempts at extraction and quantification of DNA suggest that none is present, small numbers of DNA molecules may survive in the sample. For

PeerJ reviewing PDF | (2017:10:21417:2:1:CHECK 16 Feb 2018) 
417 questions of simple species identification involving old and highly degraded samples, it may 418 only be necessary to obtain limited data - even a few hundred base pairs of mitochondrial data

419

420

421

422

423

424

425

426

427

428

429

430

431

432

433

434

435

436

437

438

439

440

441

442

443

444

445

446

447

448

449

450

451

452

453

454

455

456

457

458

459

460

461

462

may be sufficient to address the question. Our study shows that this is indeed possible even when initial assessments suggest that DNA in a tissue sample has been highly degraded. Obtaining data in these instances will likely require highly specialized extraction procedures such as the silica-column based extraction methodology utilized here, followed by short-fragment sequencing. Finally, we believe that the difficulty we confronted with this ethanol-fixed sample - which is consistent with the problems experienced by Ruane and Austin (2016) with their presumed alcohol-fixed samples ( 4 of 5 of which failed to sequence) - suggests that older ethanol-fixed specimens might be more problematic than formalin-fixed specimens for genomic sequencing efforts (see Handt et al. 1994 for a description of DNA hydrolysis). We note that tissue samples are routinely stored in $95 \%$ ethanol for future DNA sequencing, and that standard dogma is that old museum samples fixed in ethanol should be readily amenable to DNA sequencing, whereas formalin-fixed samples are expected to be particularly challenging for sequencing efforts (see Zimmermann et al. 2008). Thus, it would be both surprising and ironic if old museum specimens originally fixed and stored in ethanol prove to be less favorable for HTS than formalin-fixed specimens. This possibility has important implications for curatorial practices. Not only is potential hydrolytic damage cause for concern with whole fluid specimens stored in ethanol, but it could also be problematic for tissue samples stored in ethanol that are not maintained in sub-zero degree conditions. Evaporation of ethanol from tissue vials might render even modern tissue samples problematical for genetic analysis.

\section{CONCLUSIONS}

The development of HTS has revolutionized biological research by making genome-scale data readily available at a reasonable cost, even for non-model organisms. Systematists have fully embraced these advances in data acquisition for freshly sampled specimens, but are just beginning to harness HTS for the millions of fluid-preserved historical samples housed in natural history collections around the world. As we have shown here, acquiring genetic data from old museum specimens will sometimes present special challenges, but the information that can be gleaned from such specimens may be the only way to conclusively resolve previously intractable evolutionary and taxonomic questions.

\section{ACKNOWLEDGEMENTS}

The authors would like to thank Patrick Campbell and Donney Nicholson of The Natural History Museum, London for assistance processing the loan of liver tissue from the D. cristatellus holotype. Colin McCarthy and Oliver Crimmen kindly provided suggestions for locating information on A.H. Everett's Bornean collections and the history of fluid preservation at BMNH, respectively. We acknowledge Alison Devault and Jacob Enk at MYcroarray and MYreads both for advice and for generating the library preparation confirming the low yield of our initial DNA extraction. David Wake, Rauri Bowie, and members of the McGuire and Bowie labs provided valuable suggestions that improved the manuscript. JAM thanks the Economic Planning Unit of Malaysia and the Indonesian Institute of Sciences (LIPI) for providing scientific collecting permits. 
463

464

465

466

467

468

469

470

471

472

473

474

475

476

477

478

479

480

481

482

483

484

485

486

487

488

489

490

491

492

493

494

495

496

497

498

499

500

501

502

503

504

505

506

507

\section{REFERENCES}

Altschul, S. F., W. Gish, W. Miller, E. W. Myers, \& D. J. Lipman. 1990. Basic local alignment search tool. Journal of Molecular Biology 215:403-410.

Arbeli, Z. \& C. L. Fuentes, 2007. Improved purification and PCR amplification of DNA from environmental samples. FEMS Microbiology Letters 272:269-275.

Boulenger, G. A. 1900. Descriptions of new batrachians and reptiles from the Larut Hills, Perak. Annals and Magazine of Natural History Series 7, Volume 6:186-193.

Cotoras, D. D., G. G. R. Murray, J. Kapp, R. G. Gillespie, C. Griswold, W. B. Simison, R. E. Green, \& B. Shapiro. 2017. Ancient DNA resolves the history of Tetragnatha (Araneae, Tetragnathidae) spiders on Rapa Nui. Genes 8:403.

Dabney, J., M. Knapp, I. Glocke, M.-T. Gansauge, A. Weihmann, B. Nickel, C. Valdiosera, N. Garcia, S. Paabo, J.-L. Arsuaga, \& M. Meyer. 2013. Complete mitochondrial genome sequence of a Middle Pleistocene cave bear reconstructed from ultrashort DNA fragments. Proceedings of the National Academy of Sciences, 110:15758-15763.

Fulton, T. L. 2011. Setting Up an Ancient DNA Laboratory. Ancient DNA Methods and Protocols (ed. by M. Shapiro, B.; Hofreiter), pp. 1-11. Humana Press, New York.

Grandison, A. G. C. 1972. The Gunong Benom Expedition 1967. 5. Reptiles and amphibians of Gunong Benom with a description of a new species of Macrocalamus. Bulletin of the British Museum (Natural History) 23:45-101.

Günther, A. 1872. On the reptiles and amphibians of Borneo. Proceedings of the Zoological Society of London 1872:586-600.

Günther, A. 1880. An introduction to the study of fishes. Adam and Charles Black. Edinburgh. $743 \mathrm{pp}$.

Handt, O., M. Höss, M. Krings, \& S. Pääbo. 1994. Ancient DNA: methodological challenges. Experientia 50:524-529.

Huson, D. H., A. F. Auch, J. Qi, \& S. C. Schuster. 2007. MEGAN analysis of metagenomic data. Genome Research 17:377-386.

Hykin, S. M., K. Bi, \& J. A. McGuire. 2015. Fixing Formalin: A Method to Recover GenomicScale DNA Sequence Data from Formalin-Fixed Museum Specimens Using HighThroughput Sequencing. PLoS ONE 10(10): e0141579. doi:10.1371/journal.pone.0141579

Inger, R. F. 1983. Morphological and ecological variation in the flying lizards (genus $\underline{\text { Draco }}$ ). Fieldiana Zoology, New Series 18:vi+35 pp.

Kearse, M., R. Moir, A. Wilson, S. Stones-Havas, M. Cheung, S. Sturrock, S. Buxton, A. Cooper, S. Markowitz, C. Duran, T. Thierer, B. Ashton, P. L. Meintjes, \& A. J. Drummond. 2012. Geneious Basic: An integrated and extendable desktop software platform for the organization and analysis of sequence data. Bioinformatics 28:1647-1649.

Li, H. \& R. Durbin. 2009. Fast and accurate short read alignment with Burrows-Wheeler Transform. Bioinformatics, 25:1754-60. [PMID: 19451168]

Li, H., B. Handsaker, A. Wysoker, T. Fennell, J. Ruan, N. Homer, G. Marth, G. Abecasis, R. Durbin, \& 1000 Genome Project Data Processing Subgroup. 2009. The Sequence alignment/map (SAM) format and SAMtools. Bioinformatics 25, 2078-9. [PMID: 19505943] Manthey, U. 2008. Agamid Lizards of Southern Asia. Draconinae 1. Edition Chimaira, Frankfurt am Main, Germany. 
508

509

510

511

512

513

514

515

516

517

518

519

520

521

522

523

524

525

526

527

528

529

530

531

532

533

534

535

536

537

538

539

540

541

542

543

544

545

546

547

548

549

550

551
Mateiu, L., \& B. Rannala. 2008. Bayesian inference of errors in ancient DNA caused by postmortem degradation. Molecular Biology and Evolution 25:1503-1511.

McGuire, J. A. 2003. Allometric prediction of locomotor performance: An example from Southeast Asian flying lizards. American Naturalist 161:347-369.

McGuire, J. A., \& A. C. Alcala. 2000. A taxonomic revision of the flying lizards of the Philippine Islands (Iguania: Agamidae: Draco), with a description of a new species. Herpetological Monographs 14:81-138.

McGuire, J. A., \& B. H. Kiew. 2001. Phylogenetic systematics of Southeast Asian flying lizards (Iguania: Agamidae: Draco) as inferred from mitochondrial DNA sequence data. Biological Journal of the Linnean Society 72:203-229.

McGuire, J. A., R. M. Brown, Mumpuni, A. Riyanto, \& N. Andayani. 2007. The flying lizards of the Draco lineatus group (Squamata: Iguania: Agamidae): A taxonomic revision with descriptions of two new species. Herpetological Monographs 21:180-213.

Molak, M., \& S. Y. W. Ho. 2011. Evaluating the impact of post-mortem damage in ancient DNA: A theoretical approach. Journal of Molecular Evolution 73:244-255.

Meyer, M. \& M. Kircher. 2010. Illumina sequencing library preparation for highly multiplexed target capture and sequencing. Cold Spring Harbor Protocols, 5, 1-11.

Musters, C. J. M. 1983. Taxonomy of the genus Draco L. (Agamidae, Lacertilia, Reptilia). Zoologische Verhandelingen 199:1-120 + 4 plates.

de Rooij, N. 1915. The Reptiles of the Indo-Australian Archipelago. I. Lacertilia, Chelonia, Emydosauria. E. J. Brill Ltd., Leiden.

Palkopoulou, E., S. Mallick, P. Skoglund, J. Enk, N. Rohland, H. Li, A. Omrak, S. Vartanyan, H. Poinar, A. Götherström, D. Reich, \& L. Dalén. 2015. Complete genomes reveal signatures of demographic and genetic declines in the woolly mammoth. Current Biology 25:1395-1400.

Prüfer, K., F. Racimo, N. Patterson, F. Jay, S. Sankararaman, S. Sawyer, A. Heinze, G. Renaud, P. H. Sudmant, C. de Filippo, H. Li, S. Mallick, M. Dannemann, Q. Fu1, M. Kircher, M. Kuhlwilm, M. Lachmann, M. Meyer, M. Ongyerth, M. Siebauer, C. Theunert, A. Tandon, P. Moorjani, J. Pickrell, J. C. Mullikin, S. H. Vohr, R. E. Green, I. Hellmann, P. L. F. Johnson, H. Blanche, H. Cann, J. O. Kitzman, J. Shendure, E. E. Eichler, E. S. Lein, T. E. Bakken, L. V. Golovanova, V. B. Doronichev, M. V. Shunkov, A. P. Derevianko, B. Viola, M. Slatkin, D. Reich, Janet Kelso, \& S. Pääbo. 2014. The complete genome sequence of a Neandertal from the Altai Mountains. Nature 505(7481):43-49.

Ruane, S., \& C. C. Austin. 2016. Phylogenomics using formalin-fixed and 100+ year-old intractable natural history specimens. Molecular Ecology Resources doi:10.1111/17550998.12655.

Swofford, D. L. 2002. PAUP*. Phylogenetic Analysis Using Parsimony (*and Other Methods). Version 4. Sinauer Associates, Sunderland, Massachussetts.

Tin M. M.-Y., E. P. Economo, \& A. S. Mikheyev. 2014. Sequencing Degraded DNA from NonDestructively Sampled Museum Specimens for RAD-Tagging and Low-Coverage Shotgun Phylogenetics. PLoS ONE 9(5): e96793. doi:10.1371/journal.pone.0096793.

Uetz, P. 2006. The EMBL Reptile Database CD-ROM edition, March 2006.

Zimmermann, J., M. Hajibabaei, D. C. Blackburn, J. Hanken, E. Cantin, J. Posfai \& T. C Evans, Jr. 2008. DNA damage in preserved specimensand tissue samples: a molecular assessment. Frontiers in Zoology 5:18. 


\section{Table $\mathbf{1}$ (on next page)}

Numbers of base substitutions and sequence divergence values between the Draco cristatellus holotype and 14 exemplars representing the $D$. fimbriatus group.

Base pair differences between the Draco cristatellus holotype and each of $14 \mathrm{D}$. fimbriatus group samples for six mitochondrial genes. For ND2, the data used for comparisons were generated using standard Sanger sequencing. For all other genes, the data were derived from exome-capture off-target sequences. Mean sequence divergence values relative to the holotype are provided for each gene for each species. The final column indicates the total number of base changes and percentage divergences from the holotype, with the caveat that these summary values do not account for the unique evolutionary rates that typify each of these mitochondrial genes. 
cristatellus TNHC 56763

punctatus Borneo TNHC 56766

punctatus Borneo TNHC 56764

punctatus Malay Pen. LSUHC 5617

punctatus Mentawai MVZ 270632

punctatus Batu Ids MVZ 270636

punctatus Sumatra MVZ 270835

punctatus Banyak Ids MVZ 270829

fimbriatus Mal Pen TNHC 57954

fimbriatus Mal Pen TNHC 58565

fimbriatus Sumatra MZB Lace.14276

fimbriatus Sumatra MVZ 239473

hennigi JAM 2065 LSUMZ 81446

hennigi JAM 2066 LSUMZ 81447
COX III

contig3a

$0 / 43(0 \%)$

$5 / 43(11.6 \%)$

$4 / 43(9.3 \%)$

$4 / 43(9.3 \%)$

$4 / 43(9.3 \%)$

$4 / 43(9.3 \%)$

$4 / 43(9.3 \%)$

$4 / 43(9.3 \%)$

$\mathrm{COI}$

contig12a

$1 / 79(1.3 \%)$

$7 / 79(8.9 \%)$

$6 / 79(7.6 \%)$

$10 / 79(12.7 \%)$

$10 / 79(12.7 \%)$

9/79 (11.4\%)

0/79 (12.7\%)

$7 / 71(9.9 \%)$

$10 / 79(12.7 \%)$

9/79 (11.4\%)

9/79 (11.4\%)

$11 / 79(13.9 \%)$

$11 / 79(13.9 \%)$
COI

contig12b

$1 / 81(1.2 \%)$

$7 / 69(10.1 \%)$

8/81 (9.9\%)

$7 / 81(8.6 \%)$

$7 / 81(8.6 \%)$

$7 / 81(8.6 \%)$

$7 / 81(8.6 \%)$

$7 / 81(8.6 \%)$

$7 / 80(8.8 \%)$

$5 / 56(8.9 \%)$

$8 / 81(9.9 \%)$

(1)
ND4L

contig13

$0 / 79(0 \%)$

$10 / 69(14.5 \%)$

$10 / 79(12.7 \%)$

$12 / 79(15.2 \%)$

$12 / 79(15.2 \%)$

$9 / 59(15.3 \%)$

9/79 (11.4\%)

$8 / 79(10.1 \%)$

$8 / 79(10.1 \%)$

$8 / 79(10.1 \%)$

$8 / 79(10.1 \%)$

\section{ND5}

contig15

0/64 (0\%)

(2)

$12 / 64(18.8 \%)$

$12 / 64(18.8 \%)$

$11 / 64(17.2 \%)$

$12 / 64(18.8 \%)$

$13 / 64$ (20.3\%)
ND2

Sanger

$3 / 183(1.6 \%)$

$30 / 183(16.4 \%)$

$28 / 183(15.3 \%)$

$32 / 183(17.5 \%)$

$37 / 183(20.2 \%)$

$35 / 183(19.1 \%)$

$35 / 183(19.1 \%)$

$37 / 183(20.2 \%)$

29/183 (15.8\%)

$32 / 183(17.5 \%)$

23/183(12.6\%)

23/183 (12.6\%)

\section{Total (\%)}

$5 / 547(0.9 \%)$ $44 / 331(13.3 \%)$ $52 / 412(12.6 \%)$

$47 / 305(15.4 \%)$

$70 / 465(15.1 \%)$

$68 / 465(14.6 \%)$

$66 / 465(14.2 \%)$

67/465 (14.4\%)

68/521 (13.1\%)

$42 / 345(12.2 \%)$

$54 / 397$ (13.6\%)

$36 / 303(11.9 \%)$

$54 / 405(13.3 \%)$

$55 / 405(13.6 \%)$ 


\section{Figure $\mathbf{1}$ (on next page)}

Phylogenetic tree for the Draco fimbriatus group including the $D$. cristatellus holotype.

Phylogenetic tree for the Draco fimbriatus group based on a parsimony analysis of the complete mitochondrial ND2 gene (1032 bp). The D. cristatellus holotype includes $183 \mathrm{bp}$ of sequence data. Only two of 28 available $D$. maculatus samples were included to simplify the image. Non-parametric bootstrap values (1000 replicates) are superimposed on the single most parsimonious phylogram for select clades. The photo in the bottom left is Draco punctatus. Photo: Jimmy A. McGuire 


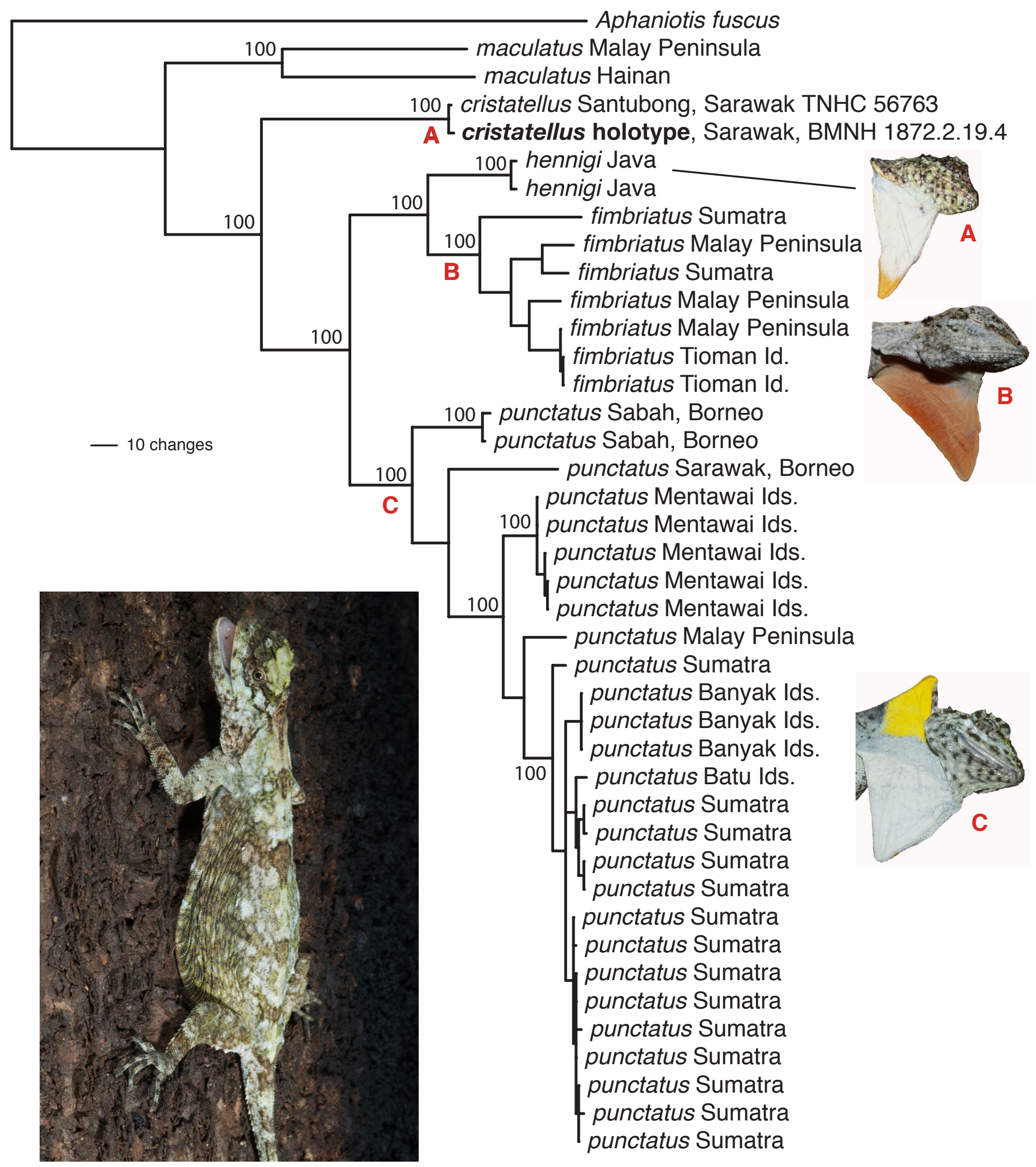

